\title{
BINARY POST-AGB STARS
}

\author{
L.B.F.M. WATERS
}

Astronomical Institute, University of Amsterdam

AND

C. WAELKENS AND H. VAN WINCKEL

Instituut voor Sterrenkunde, Katholieke Universiteit Leuven

\section{Introduction}

Low and intermediate mass stars leave the Asymptotic Giant Branch (AGB) when the mass in their $\mathrm{H}$-rich envelope is less than about $0.01 \mathrm{M}_{\odot}$, and the high mass loss drops several orders of magnitude. The central star rapidly evolves to the left part of the HR diagram along a track of constant luminosity (e.g. Schönberner 1983). In principle the evolution of the central star to higher $\mathrm{T}_{\text {eff }}$ and the expansion and cooling of the AGB remnant are easy to calculate. In practice several complicating factors arise which make it much more difficult to predict the morphology and properties of postAGB stars, such as binarity, post-AGB mass loss and aspherical AGB mass loss. Binarity of post-AGB stars affects the morphology of the circumstellar environment, and it affects evolutionary timescales and surface chemical abundances of the components in the system. This review discusses some properties of binary post-AGB stars.

\section{Hot Dust and Binarity}

The IRAS Point Source Catalogue (PSC) has been used extensively to search for new post-AGB candidates (e.g. van der Veen et al. 1989; Oudmaijer et al. 1992; Hu et al. 1993). Follow-up studies of post-AGB candidates found by cross-correlating optical catalogues with the IRAS PSC (Oudmaijer et al. 1992) showed that a significant fraction of the A, F and $\mathrm{G}$ type supergiants with cool dust shells $\left(\mathrm{T}_{\text {dust }} 100-200 \mathrm{~K}\right.$ ) found this way also have a hot dust component $\left(\mathrm{T}_{\text {dust }} 500-1300 \mathrm{~K}\right)$. Subsequent radial velocity measurements revealed a tight correlation between the occurrence of 


\section{Waters}

hot dust and binarity (Waters et al. 1990; Van Winckel et al. 1997). Perhaps the most famous example found so far is the central star of the Red Rectangle nebula, HD 44179 (Van Winckel et al. 1993). Several binaries with very wide orbits were found without hot dust (e.g. HD 179821; Van Winckel, in preparation). Among the RV Tau variables, four were found to be binaries: U Mon (Pollard, 1995), AC Her, HD 131356 (Van Winckel et al. in preparation), and HD 52961 (Waelkens et al. 1992), with orbital periods of $2500,1230,1150$, and 1330 days respectively. In addition, the W Vir star ST Pup shows RV variations consistent with binary motion (Gonzalez \& Wallerstein 1996).

If it is assumed that the hot dust is in radiative equilibrium with the radiation from the central star, the hot dust must be very close to the central star. This implies either a significant post-AGB mass loss, or the dust is not in an outflow but stored somewhere in the binary system. Other indicators of post-AGB mass loss, such as $\mathrm{H} \alpha$ line emission, suggest a present-day mass loss rate which is lower than that derived from the hot dust component (Waters et al. 1993). The conclusion then is that the dust is stored in the system. The temperature of the dust is in most cases consistent with a location (just) outside the binary, probably in a circum-binary disc. The origin of the disc is unclear, but may be related to the AGB mass loss.

The nature of the companions of binary post-AGB stars is unknown. The mass function for many systems is compatible with a low mass main sequence star, or with a white dwarf. In HR 4049, a search for X-ray emission, which may indicate the presence of a white dwarf, was negative (Verbunt, private communication). This points to a main sequence star companion for HR 4049.

\section{Surface chemical abundances}

Abundance studies of optically bright post-AGB stars reveal a rather confusing picture. For single post-AGB stars, there is a large scatter in abundance patterns (e.g. Luck et al. 1990; van Winckel 1995; Reddy et al. 1996). The general trend however is that CNO processed material appears at the surface, but sometimes the pattern is indicative of the first dredgeup rather than the 3rd dredge-up. Only very few stars in the sample are C-rich and show s-process enhancements pointing to efficient dredge-up (e.g. HD 56126, Klochkova 1995; HD 187885; van Winckel et al. 1996). In addition, stars with very similar $\mathrm{T}_{\text {eff }}, \log \mathrm{g}$ and iron content show very different 3rd dredge-up efficiencies.

The binary post-AGB stars can be divided into two groups: stars with abundance patterns similar to single stars (e.g. 89 Her; Luck et al. 1990), and a group of very metal deficient objects (e.g. Lambert et al. 1988; Van 


\section{BINARY POST-AGB STARS}

Winckel et al. 1995), with $[\mathrm{Fe} / \mathrm{H}]$ between -3 and -5 . It was pointed out by Venn \& Lambert (1990) that the abundance patterns in these extreme cases resemble those of the depletion of gas-phase elements in the ISM, i.e. strong underabundance of elements as $\mathrm{Mg}, \mathrm{Fe}, \mathrm{Ti}$, and $\mathrm{Cr}$, while $\mathrm{C}$, $\mathrm{N}, \mathrm{O}$, and $\mathrm{S}$ are solar. The detection of $\mathrm{Zn}$ with roughly solar abundance in HD 52961 (Van Winckel et al. 1992) and the Red Rectangle (Waelkens et al. 1996) confirmed this depletion scenario. We will return to the metal deficient binaries below.

\section{Circumstellar dust}

The composition of circumstellar dust in binary post-AGB stars can be both oxygen-rich (e.g. $89 \mathrm{Her}$ ) and carbon-rich (e.g. HR 4049). Interestingly, the photospheric $\mathrm{C} / \mathrm{O}$ ratio does not always match the observed chemistry in the circumstellar dust. For instance, in HR 4049 the $\mathrm{C} / \mathrm{O}$ ratio is about 1 but the shell has prominent emission from C-rich grains (Buss et al. 1993; Molster et al. 1996). All objects with extreme metal deficiencies have Crich dust. In HD 213985 the detection of $\mathrm{CH}^{+}$absorption in the optical spectrum clearly points to a C-rich envelope (Waelkens et al. 1995a).

The extinction properties of the dust at optical and UV wavelengths show a large range in behaviour. The UV extinction law of HR 4049 does not show the 2175 A bump (Waters et al. 1988; Buss et al. 1989), while in HD 213985 there is a prominent bump, shifted to about $2300 \AA$ (Buss et al. 1989). This shift indicates that the dust probably consists of small graphite grains, perhaps similar to those seen in R CrB stars (Hecht et al., 1984). The dust shell in HD 213985 consists of two components, a high temperature component (similar to HR 4049) and a much cooler component, which probably represents the AGB remnant.

The extinction towards HR 4049 and HD 213985 varies in phase with the binary motion. This variability can be explained if the absorbing dust is in a (thick) disc around the binary system, and the system is viewed at an intermediate inclination angle (Waelkens et al. 1991).

\section{The Red Rectangle: a case study}

The central star of the Red Rectangle nebula, HD 44179, belongs to the small class of extremely metal deficient binary post-AGB stars. The object is famous for its remarkable optical reflection nebulosity with a pronounced $\mathrm{X}$ shape (Cohen et al. 1975). Imaging at high angular resolution has shown that the central star is obscured from view in the optical, and that only scattered light from above and below what appears to be a disc reaches the observer (Roddier et al. 1995; Bond et al., and Osterbart et al., these proceedings). Given the symmetry of the nebula, which suggests that we 


\section{Waters}

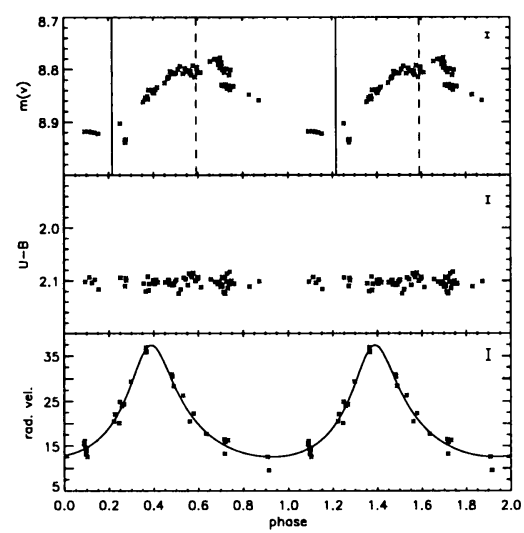

Figure 1. Geneva V and U-B photometry (top panels) plotted as a function of orbital phase and compared to the radial velocity curve (lower panel). Note the variations in $\mathrm{V}$ and the lack of variations in U-B

view the system edge-on, it is remarkable that radial velocity variations are observed in the scattered light. The object also is photometrically variable with the same period as the orbital one; no colour variations are seen (figure 1).

The photometric variations and their correlation with orbital motion can be explained in a geometric model of the binary system, in which the bulk of the scattering particles (dust grains) are viewed at an angle of about 55 degrees with respect to the binary system (figure 2). The photometric variations are the result of the change in scattering angle as the bright postAGB star moves in its orbit. This model also explains the lack of colour variations, since a change in scattering angle does not affect colours.

The nebular material is carbon-rich, as evidenced by the prominent UIR bands (Russell et al. 1978), that are usually attributed to small C-rich dust grains. Recently, Jura el al. (1997) found evidence for the presence of large dust grains. Clearly a very large range of particle sizes must be present in the nebula. Waelkens et al. (1992) detected narrow emission lines in the optical spectrum, that were identified by Balm \& Jura (1992) as due to $\mathrm{CH}^{+}$. Jura et al. (1995) and Bachiller et al. (1988) detected CO millimeter line emission from the nebula, and found a very weak line compared to the $60 \mu \mathrm{m}$ dust emission. This suggests that the gas to dust ratio in the nebula is much lower than usually found.

The abundance pattern of the metals in the photosphere of the central star, HD 44179, shows a depletion pattern similar to that seen in the interstellar medium. This points to dust formation and separation of gas and dust, leaving the gas devoid of metals. This process must have occurred in the circumstellar environment, since dust formation in stellar photospheres 


\section{BINARY POST-AGB STARS}

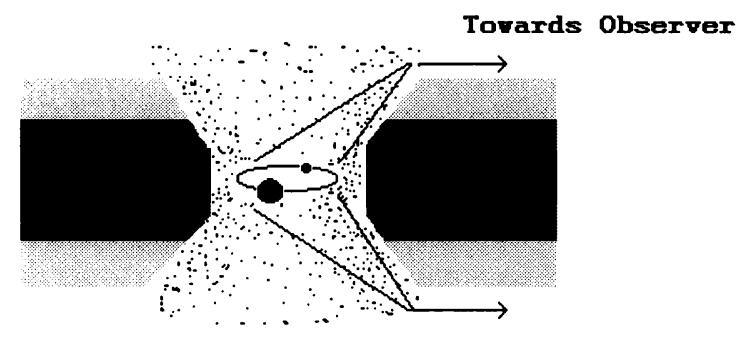

Figure 2. A geometrical model for the Red Rectangle nebula and its central binary (Van Winckel et al. 1996)

is unlikely. It is possible that the material located in the circum-binary disc can partially re-accrete onto the star. The radiation pressure exerted on the dust grains would prevent the grains from re-accreting, while the gas experiences no significant radiation pressure and can accrete. This mechanism can qualitatively explain the surface abundances and the low $\mathrm{CO}$ emission in the nebula. Numerical simulations of particle flows in a circumbinary disc show that accretion of material can indeed occur (Artymowicz \& Lubow 1996).

\section{An evolutionary scenario}

A remarkable aspect of the orbital solutions found for several binary postAGB stars is the large eccentricity (up to e $=0.38$; Van Winckel et al. 1995 ) in combination with a relatively short orbital period (several 100 days). These short orbital periods point to strong interaction between the two components during the AGB phase (common envelope phase), but clearly the orbits have not circularized. This puzzling contradiction suggests that the evolution of the AGB star in these systems may have been highly non-standard, avoiding a common envelope phase. Alternately, the orbital eccentricity may have developed after the AGB through tidal interaction with a (massive) circum-binary disc. Such a mechanism is also invoked to explain the sometimes large eccentricities in young binaries.

The metal deficient post-AGB binaries have orbital elements that are quite similar to those found in Barium dwarfs and giants (McClure et al. 1980; Jorissen \& Mayor 1988), and in S stars without Tc (Jorissen \& Mayor 1992). The barium stars and their population II counterparts (the CH stars) are all in wide binary systems, often with non-zero eccentricities. The mass functions of the $\mathrm{Ba}$ stars are consistent with a very narrow distribution of companion masses around $0.6 \mathrm{M}_{\odot}$, suggesting a white dwarf nature 


\section{Waters}

of the companion. The $\mathrm{Ba}$ enrichment in these stars is probably due to mass transfer during the AGB evolution of the current white dwarf. The metal deficient post-AGB binaries may be the progenitors of the barium dwarf and giant stars. If this is the case, the (unseen) companions of the metal deficient post-AGB stars should already be enriched in s process elements. An evolutionary scenario could then be: MS+MS, MS+Red Giant, MS+AGB, Ba-dwarf+Fe-deficient PAGB, Ba-dwarf+WD, Ba-giant+WD, S-star+WD, WD+WD.

\section{References}

Artymowicz, P., Lubow, S.H.: 1996, ApJ 467, L77

Bachiller, R., Gomez-Gonzalez, J., Bujarrabal, V., Martin-Pintado, J.: 1988, A\&A 196, L5

Balm, S.P., Jura, M.: 1992, A\&A 261, L25

Buss, R.H., Snow, T.P., Lamers, H.J.G.L.M.: 1989, ApJ 347, 977

Buss, R.H., Tielens, A.G.G.M., Cohen, M., Werner, M.W., Bregman, J.D., Witteborn, F.C.: 1993, ApJ 415, 250

Cohen, M., Anderson, C.M., Cowley, A., Coyne, G.V., Fawley, W.M., Gull, T.R., Harlan, E.A., Herbig, G.H., Holden, F., Hudson, H.S., Jakoubek, R.O., Johnson, H.M., Merill, K.M., Schiffer, F.H., Soifer, B.T., Zuckerman, B.: 1975, ApJ 196, 179

Hecht, J.H., Holm, A.V., Donn, B., Wu, C.-C.: 1984, ApJ 280, 228

Hu, J.Y., Slijkhuis, S., de Jong, T., Jiang, B.W.: 1993, A\&AS 100, 413

Jorissen, A., Mayor, M.: 1988, A\&A 198, 187

Jorissen, A., Mayor, M.: 1992, A\&A 260, 115

Jura, M., Balm, S.P., Kahane, C.: 1995, ApJ 453, 721

Jura, M., Turner, J., Balm, S.P.: 1997, ApJ 474, 741

Klochkova, V.G.: 1995, MNRAS 272, 710

Luck, R.E., Bond, H.E., Lambert, D.L.: 1990, ApJ 357, 188

McClure, R.D., Fletcher, J.M., Nemec, J.M.: 1980, ApJ 238, L35

Oudmaijer, R.D., van der Veen, W.E.C.J., Waters, L.B.F.M., Trams, N.R., Waelkens, C., Engelsman, E.: 1992, A\&AS 96, 625

Pollard, K.R.: 1995, in A.S.P. series vol. 83, eds. R.S. Stobie \& P.A. Whitelock, p. 409

Reddy, B.E., Parthasarathy, M., Sivarani, T.: 1996, A\&A 313, 191

Roddier, F., Roddier, C., Graves, J.E., Northcott, M.J.: 1995, ApJ 443, 249

Russell, R.W., Soifer, B.T., Willner, S.P.: 1978, ApJ 220, 568

Van der Veen, W.E.C.J., Habing, H.J., Geballe, T.R.: 1989, A\&A 226, 108

Van Winckel, H., Mathis, J.S., Waelkens, C.: 1992, Nature 356, 500

Van Winckel, H.: 1995, thesis, University of Leuven

Van Winckel, H., Waelkens, C., Waters, L.B.F.M.: 1995, A\&A 293, L25

Van Winckel, H., Waelkens, C., Waters, L.B.F.M.: 1996, A\&A 306, L37

Venn, Lambert, D.L.: 1990, ApJ 363, 234

Waelkens, C., Lamers, H.J.G.L.M., Waters, L.B.F.M., Rufener, F., Trams, N.R., Trams, N.R., Le Bertre, T., Ferlet, R., Vidal-Madjar, A.: 1991, A\&A 242, 433

Waelkens, C., Van Winckel, H., Trams, N.R., Waters, L.B.F.M.: 1992, A\&A 256, L15

Waelkens, C., Waters, L.B.F.M., Van Winckel, H., Daems, K.: 1995a, in proc. of Circumstellar Matter, eds. G.D. Watt \& P.M. Williams, Astrophys. Sp. Sc. 224, 357

Waelkens, C., Van Winckel, H., Waters, L.B.F.M., Bakker, E.J.: 1996, A\&A 314, L17

Waters, L.B.F.M., Lamers, H.J.G.L.M., Snow, T.P., et al.: 1989, A\&A 211, 208

Waters, L.B.F.M., Waelkens, C., Mayor, M., Trams, N.R.: 1993, A\&A 269, 242 